\title{
On-Line Modeling of AUV's Maneuvering Motion Based on Sliding Window SVM
}

\author{
Pang Yongjie \\ State Key Laboratory of Autonomous Underwater \\ Vehicle \\ Harbin Engineering University \\ Harbin, China \\ e-mail: pangyongjie@hrbeu.edu.cn
}

\section{Hu Zhonghui}

State Key Laboratory of Autonomous Underwater

Vehicle

Harbin Engineering University

Harbin, China

e-mail: huhuige369369@qq.com

\author{
Zhang Guocheng \\ State Key Laboratory of Autonomous Underwater \\ Vehicle \\ Harbin Engineering University \\ Harbin, China \\ e-mail: zhangguocheng168@126.com \\ Sun Yushan \\ State Key Laboratory of Autonomous Underwater \\ Vehicle \\ Harbin Engineering University \\ Harbin, China \\ e-mail: sunyushan@hrbeu.edu.cn
}

\begin{abstract}
In order to solve the problem of on-line modeling of $A U V^{\prime}$ ' Maneuvering Motion, considering that the sample space expends in the parametric identification, a learning algorithm for least squares support vector machines (LS-SVM) based on sliding window is deduced. The method improves the identification results of SVM and has the merits such as computing fast and cost less storage space. Firstly, based on the theory of sliding window, a data region which is sliding with time is built to restrain matrix dimension expending in the parametric identification. Then, the parameters of a certain $A U V$ are identified on-line based on the free running model test. And the veracity of the parameters identified is validated by using the system simulation. The results demonstrate that the method which can identify the parameters effectively is feasible and effective.
\end{abstract}

Keywords- least squares support vector machines; sliding window; parametric identification; free Running model test

\section{INTRODUCTION}

Maneuverability is one of the most important hydrodynamic performances of AUV, which has a close relationship with navigation safety ${ }^{[1]}$. An accurate modeling of AUV maneuvering motion has great significance to the maneuverability prediction. And accurately determining the hydrodynamic derivatives is vital. In recent years, a novel method of artificial intelligence algorithm, Support Vector Machines (SVM), is widely used in the area of system identification. Zou and his research group identified the hydrodynamic derivatives and maneuvering indices in the mathematical models of maneuvering motion by using $\mathrm{SVM}^{[2-4]}$. Luo ${ }^{[5]}$ has made a lot of efforts on modeling of ship maneuvering by using SVM. Xu ${ }^{[6]}$, from Shanghai Jiao Tong

Supported by "the Fundamental Research Funds for the Central University" (HEUCF1321005)( HEUCF1321006)
University, used on-line SVM to identify the hydrodynamic parameters of AUV. The literature [7] studied on the on-line modeling of ship maneuvering motion by using incremental least squares support vector machines (LS-SVM). Although incremental LS-SVM can be used as an on-line algorithm, it has two disadvantages. One is that it considers all the samples and hasn't oblivion mechanism which makes the algorithm cost much computing time and storage space. The other one is the complexity of calculation. With the number of training samples increasing, the complexity of calculation is too great to complete the on-line training. LS-SVM based on sliding window is proposed to solve the problem. It fully utilizes the historical training results so that the calculating complexity is decreased and the calculating efficiency is improved. An AUV's hydrodynamic coefficients are on-line identified by using sliding window LS-SVM. The simulation results demonstrate that the method is feasible and effective. Compared with incremental LS-SVM, sliding window LSSVM has faster computing speed and costs less storage space. It is more suitable to the on-line parametric identification of AUV's maneuvering motion.

\section{LS-SVM}

J.A.K. Suykens ${ }^{[8]}$ proposed least squares support vector machine(LS-SVM) method based on standard SVM, which used the sum of error's squares as loss function. It changes inequality constraints into equality constraints and the optimization problem into a linear equation, which improves the computing speed, the practicality of standard SVM and reduces the computation storage space effectively.

The sample set is described as

$$
\left\{\left(x_{1}, y_{1}\right),\left(x_{2}, y_{2}\right), \ldots,\left(x_{l}, y_{l}\right)\right\}
$$

Where, $x_{i} \in R^{n}, y_{i} \in R, l$ is the total number of sample set. LS-SVM regression problem can be described as follows: 


$$
\begin{gathered}
\min _{w, b, \xi} J(w, \xi)=\frac{1}{2} w^{T} w+\frac{1}{2} C \sum_{i=1}^{l} \xi_{i}^{2} \\
\text { s.t. } y_{i}=w \cdot \varphi\left(x_{i}\right)+b+\xi_{i}
\end{gathered}
$$

Where, $\xi_{i}$ is regression error, $C$ is rule factor.

Lagrange function is defined for objective function and constraint conditions,

$$
\begin{aligned}
& L(w, b, \xi, \alpha)=\frac{1}{2} w^{T} w+\frac{1}{2} C \sum_{i=1}^{l} \xi_{i}^{2}- \\
& \sum_{i=1}^{l} \alpha_{i}\left(w \cdot \varphi\left(x_{i}\right)+b+\xi_{i}-y_{i}\right)
\end{aligned}
$$

Where, $\alpha_{i}$ is Lagrange multiplier. Partial derivative with respect to $w, b, \xi$ and $\alpha$ gives:

$$
\left\{\begin{array}{l}
\frac{\partial L}{\partial w}=0 \rightarrow w=\sum_{i=1}^{l} \alpha_{i} \varphi\left(x_{i}\right)=0 \\
\frac{\partial L}{\partial b}=0 \rightarrow \sum_{i=1}^{l} \alpha_{i}=0 \\
\frac{\partial L}{\partial \xi_{i}}=0 \rightarrow \alpha_{i}=C \xi_{i} \\
\frac{\partial L}{\partial \alpha_{i}}=0 \rightarrow w \cdot \varphi\left(x_{i}\right)+b+\xi_{i}-y_{i}=0
\end{array}\right.
$$

Substituting the first and second formulas into the forth, subject to third, gives:

$$
\left[\begin{array}{cc}
0 & e \\
e^{T} & \Omega+C^{-1} I
\end{array}\right]\left[\begin{array}{l}
b \\
\alpha
\end{array}\right]=\left[\begin{array}{l}
0 \\
y
\end{array}\right]
$$

Where, $y=\left(y_{1}, y_{2}, \ldots, y_{l}\right)^{T}, e=(1,1, \ldots, 1)^{T}$, $\alpha=\left(\alpha_{1}, \alpha_{2}, \ldots, \alpha_{l}\right)^{T}, \Omega_{i j}=\left(\varphi\left(x_{i}\right) \cdot \varphi\left(x_{j}\right)\right)=K\left(x_{i}, x_{j}\right)$, $i, j=1,2, \ldots, l$. Regression estimation function can be obtained once Eq.(5) is solved,

$$
f(x)=\sum_{i=1}^{l} \alpha_{i} K\left(x, x_{i}\right)+b
$$

\section{SLIDING WINDOW LS-SVM}

With regard to sliding window LS-SVM, the sample number for identification is invariant. Along with the time updating, a new sample is added to the sample set and an old sample is reduced every time. Consequently, the sample set can be described as a function of time step $t$, i.e. $\{x(t), y(t)\}$, where, $x(t)=\left[x_{t}, x_{t+1}, \ldots, x_{t+l-1}\right]^{T}, y(t)=\left[y_{t}, y_{t+1}, \ldots, y_{t+l-1}\right]^{T}$, $l$ is the length of sliding window. In this way, the kernel function matrix $\Omega$, Lagrange multiplier $\alpha$ and offset $b$ are all functions of $t$. They can be described as follows:

$$
\begin{gathered}
\Omega_{t}(i, j)=K\left(x_{i+t-1}, x_{j+t-1}\right), i, j=1,2, \cdots, l \\
\alpha(t)=\left(\alpha_{t}, \alpha_{t+1}, \cdots, \alpha_{t+l-1}\right)^{T}, b(t)=b_{t}
\end{gathered}
$$

Then Eq.(6) can be represented as follows:

$$
y(x, t)=\sum_{i=t}^{t+l-1} \alpha(t) K\left(x, x_{i}\right)+b(t)
$$

Assuming $\Gamma(t)=\Omega_{t}+C^{-1} I$, we obtain from the result of LS-SVM:

$$
\left[\begin{array}{cc}
0 & e(t) \\
e(t)^{T} & \Gamma(t)
\end{array}\right]\left[\begin{array}{l}
b(t) \\
\alpha(t)
\end{array}\right]=\left[\begin{array}{c}
\overrightarrow{0} \\
y(t)
\end{array}\right]
$$

Assuming $U(t)=\Gamma(t)^{-1}$, solving Eq.(8), we obtain

$$
\begin{gathered}
\alpha(t)=U(t)\left[y(t)-\frac{e(t) e(t)^{T} U(t) y(t)}{e(t)^{T} U(t) e(t)}\right] \\
b(t)=\frac{e(t)^{T} U(t) y(t)}{e(t)^{T} U(t) e(t)}
\end{gathered}
$$

From Eq.(9) and Eq.(10), it is not difficult to find out that the essential question is solving $U(t)$. The kernel function matrix is $l \times l$ square matrix at the time step $t$. It gives

$$
\Gamma(t)=\left[\begin{array}{ccc}
k\left(x_{t}, x_{t}\right)+1 / C & \cdots & k\left(x_{t+l-1}, x_{t}\right) \\
\vdots & \ddots & \vdots \\
k\left(x_{t}, x_{t+l-1}\right) & \cdots & k\left(x_{t+l-1}, x_{t+l-1}\right)+1 / C
\end{array}\right]
$$

$\Gamma(t)$ can be described as partitioned matrix:

$$
\Gamma(t)=\left[\begin{array}{cc}
f(t) & F(t)^{T} \\
F(t) & W(t+1)
\end{array}\right]
$$

Where

$$
\begin{aligned}
f(t) & =k\left(x_{t}, x_{t}\right)+1 / C \\
F(t) & =\left[k\left(x_{t+1}, x_{t}\right), \ldots, k\left(x_{t+l-1}, x_{t}\right)\right]^{T} \\
W(t) & =\left[\begin{array}{ccc}
k\left(x_{t+1}, x_{t+1}\right)+1 / C & \ldots & k\left(x_{t+l-1}, x_{t+1}\right) \\
\vdots & \ddots & \vdots \\
k\left(x_{t+1}, x_{t+l-1}\right) & \cdots & k\left(x_{t+l-1}, x_{t+l-1}\right)+1 / C
\end{array}\right]
\end{aligned}
$$


At the time step $t+1$, the new sample $\left(x_{t+l}, y_{t+l}\right)$ is added and the old sample $\left(x_{t}, y_{t}\right)$ is reduced. $\Gamma(t)$ turns into $\Gamma(t+1)$ :

$$
\begin{aligned}
& \Gamma(t+1)=\Omega_{t+1}+C^{-1} I= \\
& {\left[\begin{array}{ccc}
k\left(x_{t+1}, x_{t+1}\right)+1 / C & \cdots & k\left(x_{t+l}, x_{t+1}\right) \\
\vdots & \ddots & \vdots \\
k\left(x_{t+1}, x_{t+l}\right) & \cdots & k\left(x_{t+l}, x_{t+l}\right)+1 / C
\end{array}\right]}
\end{aligned}
$$

Comparing $\Gamma(t)$ with $\Gamma(t+1), \Gamma(t+1)$ can be described as follows:

$$
\Gamma(t+1)=\left[\begin{array}{cc}
W(t) & V(t+1) \\
V(t+1)^{T} & v(t+1)
\end{array}\right]
$$

Where

$$
\begin{aligned}
& v(t+1)=k\left(x_{t+l}, x_{t+l}\right)+1 / C \\
& V(t+1)=\left[k\left(x_{t+l}, x_{t+1}\right), \ldots, k\left(x_{t+l}, x_{t+l-1}\right)\right]^{T}
\end{aligned}
$$

If $A$ can be described as partitioned matrix:

$$
A=\left[\begin{array}{cc}
A_{11} & E \\
E & d
\end{array}\right]
$$

And both $A^{-1}$ and $A_{11}^{-1}$ exist simultaneously, then it can be decomposed as follows ${ }^{[9]}$ :

$$
\begin{aligned}
& A^{-1}=\left[\begin{array}{ll}
A_{11} & E \\
E^{T} & d
\end{array}\right]=\left[\begin{array}{cc}
A_{11}^{-1} & 0 \\
0 & 0
\end{array}\right]+ \\
& {\left[\begin{array}{c}
A_{11}^{-1} E \\
-I
\end{array}\right]\left(d-E^{T} A_{11}{ }^{-1} E\right)^{-1}\left[E^{T} A_{11}^{-1}-I\right]}
\end{aligned}
$$

According to Eq.(15), $U(t)$ and $U(t+1)$ is solved:

$$
\begin{gathered}
U(t)=\Gamma(t)^{-1}=\left[\begin{array}{cc}
f(t) & F(t)^{T} \\
F(t) & W(t)
\end{array}\right]^{-1}= \\
{\left[\begin{array}{cc}
0 & 0 \\
0 & W(t)^{-1}
\end{array}\right]+r_{1}(t) z_{1}(t) r_{1}(t)^{T}} \\
U(t+1)=\Gamma(t+1)^{-1}=\left[\begin{array}{cc}
W(t) & V(t+1) \\
V(t+1)^{T} & v(t+1)
\end{array}\right]^{-1}= \\
{\left[\begin{array}{cc}
W(t)^{-1} & 0 \\
0 & 0
\end{array}\right]+r_{2}(t+1) z_{2}(t+1) r_{2}(t+1)^{T}}
\end{gathered}
$$

Where

$$
r_{1}(t)=\left[-1, \quad F(t)^{T} W(t)^{-1}\right]^{T}
$$

$$
\begin{aligned}
& z_{1}(t)=\left(f(t)-F(t)^{T} W(t)^{-1} F(t)\right)^{-1} \\
& r_{2}(t+1)=\left[\begin{array}{ll}
V(t+1)^{T} W(t)^{-1} & -1
\end{array}\right]^{T} \\
& z_{2}(t+1)=\left(v(t+1)-V(t+1)^{T} W(t)^{-1} V(t+1)\right)^{-1}
\end{aligned}
$$

Eq.(17) contains two elements, one is $W(t)^{-1}$ constituted by the data at time step $t$, and the other one is $r_{2}(t+1) z_{2}(t+1) r_{2}(t+1)^{T}$. It is the updating element constituted by the added data at time step $t+1$. It is not difficult to find out that the essential question is solving $W(t)^{-1}$. Now we are solving $W(t)^{-1}$ by using $U(t)$. Eq.(16) is rewritten as follows:

$$
\begin{aligned}
& U(t)=\left[\begin{array}{cc}
0 & 0 \\
0 & W(t)^{-1}
\end{array}\right]+ \\
& {\left[\begin{array}{cc}
\frac{1}{z_{2}(t)} & \frac{-\Gamma(t)^{T} W(t)^{-1}}{z_{2}(t)} \\
\frac{-W(t)^{-1} F(t)}{z_{2}(t)} & \frac{W(t)^{-1} F(t) F(t)^{T} W(t)^{-1}}{z_{2}(t)}
\end{array}\right]}
\end{aligned}
$$

From Eq.(18) we know, if $U(t)$ is known, we can solve $z_{2}(t)$ according to the first element of $U(t)$ 's diagonal line. Also according to the first row of $U(t)$, we can obtain

$$
P_{1}(t)=\frac{-\Gamma(t)^{T} W(t)^{-1}}{z_{2}(t)}
$$

Also according to the first column of $U(t)$, we can obtain

$$
P_{2}(t)=\frac{-W(t)^{-1} F(t)}{z_{2}(t)}
$$

Then we can obtain

$$
P_{3}(t)=\frac{W(t)^{-1} F(t) F(t)^{T} W(t)^{-1}}{z_{2}(t)}=P_{2}(t) P_{1}(t) z_{2}(t)
$$

From Eq.(16) we know, $W(t)^{-1}$ equal to the matrix that cutting the first row and first column of $U(t)$ and then reducing $P_{3}(t)$. In this way, we can solve $W(t)^{-1}$ from $U(t)$ and then obtain $U(t+1)$ according to Eq.(17). This method avoids solving the inversion of $\Gamma$ for acquiring $U(t)$ and reducing the computing complexity of learning algorithm.

Summarizing the above deducing process, the algorithm for sliding window LS-SVM can be described as follows:

Step 1: Set the initial sliding window size and parameters for system identification and $t$ must be higher than 2 . 
Step 2: Calculate $W(t)^{-1}$ or initial $W(t)^{-1}$ arbitrarily, $t \rightarrow t+1$.

Step 3: Collect new sample data and calculate $U(t)$ by using Eq.(16).

Step 4: Calculate $b(t), \alpha(t)$ and $y(x, t)$.

Step 5: Calculate $W(t)^{-1}$ by using Eq.(17).

Step 6: $t \leftarrow t+1$, goto (3).

\section{MATHEMATICAL MODEL OF AUV MANEUVERING MOTION}

This paper on-line identified the hydrodynamic derivations of Abkowitz model by using sliding window LSSVM.

Generally, we should consider all the six degrees of freedom motion of $\mathrm{AUV}^{[10]}$. However, at the weak maneuvering condition, the motion of AUV can be decomposed into two motions in the horizontal plane and in the vertical plane. Only the motion in the horizontal plane will be discussed in this paper. The horizontal motion equation can be described as ${ }^{[1],[11]}$ :

$$
\left\{\begin{array}{l}
m\left(\dot{u}-v r-y_{G} \dot{r}-x_{G} r^{2}\right)=X_{H}+X_{P} \\
m\left(\dot{v}+u r+x_{G} \dot{r}-y_{G} r^{2}\right)=Y_{H}+Y_{P} \\
I_{z} \dot{r}+m\left[x_{G}(\dot{v}+u r)-y_{G}(\dot{u}-v r)\right]=N_{H}+N_{P}
\end{array}\right.
$$

Where, $m$ is the mass of AUV, $I_{z}$ is the moment of inertia, $x_{G}, y_{G}$ are the coordinates of the gravity center, $X_{H}$, $Y_{H}, N_{H}$ are the fluid forces and moments acting on the hull, $X_{P}, Y_{P}, N_{P}$ are the forces and moments of propeller, $u, v$, $r$ are the components of the speed and the yaw rate of AUV, $\dot{u}, \dot{v}, \dot{r}$ are the components of the acceleration and the angular acceleration.

The fluid forces and moments $X_{H}, Y_{H}, N_{H}$ are expanded with Taylor series. Under the weak maneuvering condition, the longitudinal motion equation degenerates into an equilibrium equation with the balance between thrust and resistance. It is decoupled from the equation system and can be treated separately. Thus the motion equation of horizontal plane can be reconstructed as

$$
\begin{aligned}
& {\left[\begin{array}{cc}
m-Y_{\dot{v}} & m x_{G}-Y_{\dot{r}} \\
m x_{G}-N_{\dot{v}} & I_{Z}-N_{\dot{r}}
\end{array}\right]\left[\begin{array}{c}
\dot{v} \\
\dot{r}
\end{array}\right]=\left[\begin{array}{cc}
Y_{v} & Y_{r}-m U \\
N_{v} & N_{r}-m x_{G} U
\end{array}\right]\left[\begin{array}{l}
v \\
r
\end{array}\right]+} \\
& {\left[\begin{array}{ccccc}
1 & 0 & Y_{v|v|} & Y_{r|r|} & Y_{v|r|} \\
0 & 1 & N_{v|v|} & N_{r|r|} & N_{v|r|}
\end{array}\right]\left[\begin{array}{c}
Y_{P} \\
N_{P} \\
v|v| \\
r|r| \\
v|r|
\end{array}\right]}
\end{aligned}
$$

According to the principle of parameter identifiability, inertial coefficients can be obtained by theoretical calculation or captive model tests rather than by system identification. Therefore, there are total ten hydrodynamic coefficients to be identified.

\section{ON-LINE PARAMETRIC IDENTIFICATION}

Horizontal $10^{\circ} / 10^{\circ}$ zigzag test is designed to identify the Abkowitz model. The sample period is $0.5 \mathrm{~s}$ and the sampling number is 900. Linear Kernel function is adopted. The rule factor is set as 500 and the length of sliding window is 150 . Then hydrodynamic derivatives are identified varying with window sliding. The variable derivatives are shown in Fig.1.
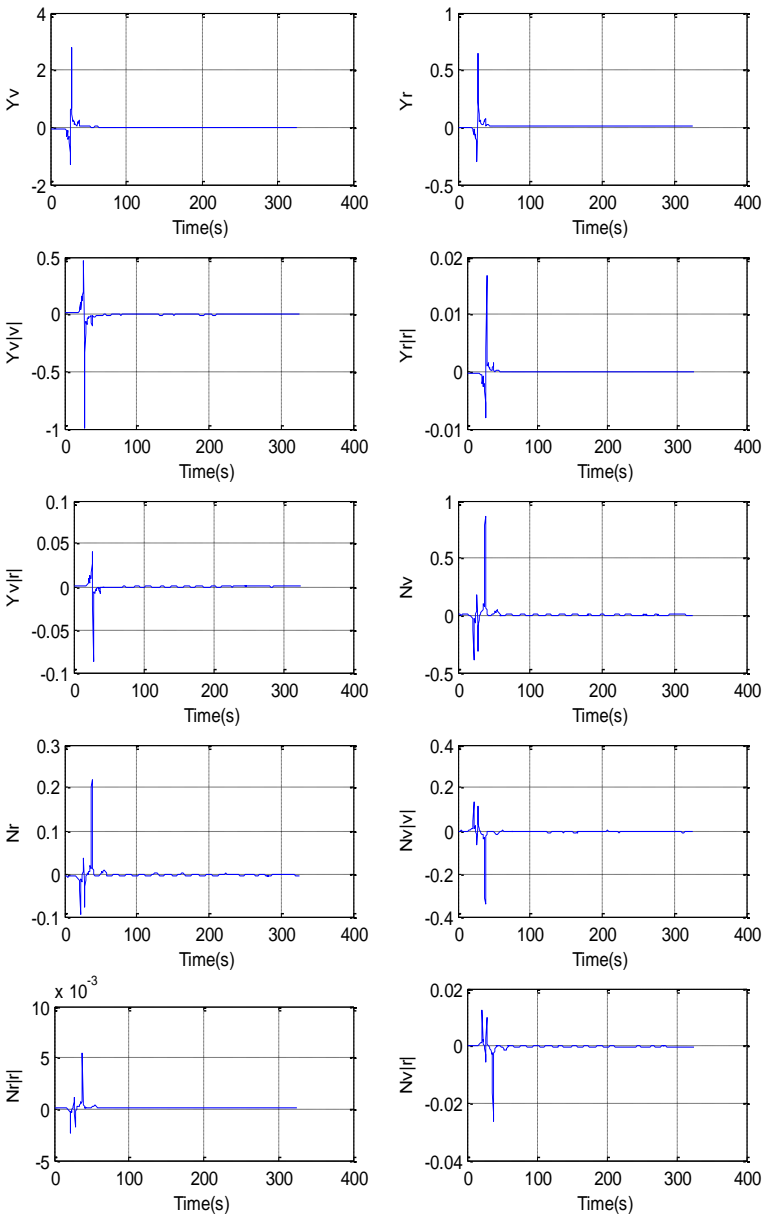

Figure 1. Time histories of the hydrodynamic coefficients

From Fig.1, it is easy to find that the derivatives are close to a fixed value gradually. The identification result is shown in Table I .

TABLE I. IDENTIFICATION RESULTS OF HYDRODYNAMIC COEFFICIENTS

\begin{tabular}{|c|c|c|c|c|}
\hline$Y_{v}^{\prime}$ & $Y_{r}^{\prime}$ & $Y_{v|v|}^{\prime}$ & $Y_{r|r|}^{\prime}$ & $Y_{v|r|}^{\prime}$ \\
\hline $2.45 \mathrm{E}-2$ & $4.36 \mathrm{E}-2$ & $-5.66 \mathrm{E}-3$ & $-4.49 \mathrm{E}-4$ & $6.83 \mathrm{E}-4$ \\
\hline$N_{v}^{\prime}$ & $N_{r}^{\prime}$ & $N_{v|v|}^{\prime}$ & $N_{r|r|}^{\prime}$ & $N_{v|r|}^{\prime}$ \\
\hline $6.25 \mathrm{E}-3$ & $-1.43 \mathrm{E}-2$ & $-1.22 \mathrm{E}-2$ & $3.14 \mathrm{E}-4$ & $-1.19 \mathrm{E}-3$ \\
\hline
\end{tabular}


This paper demonstrates the veracity of the identified hydrodynamic coefficients by using system simulation. The results of the simulation are compared with the experimental results as shown in Fig.2 and Fig.3.

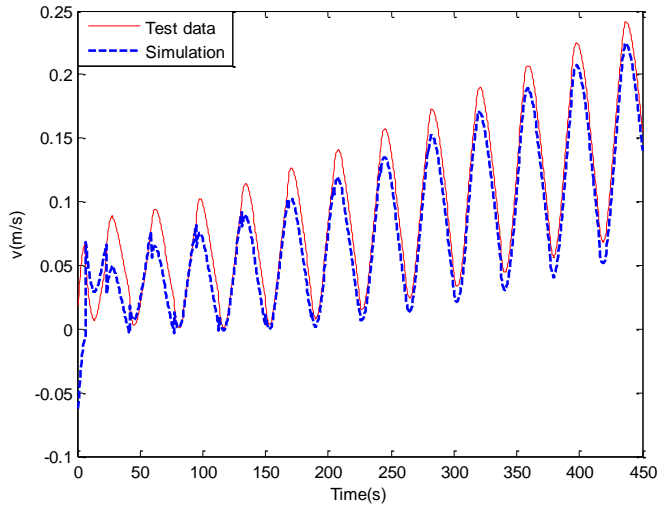

Figure 2. Time histories of the transverse speed

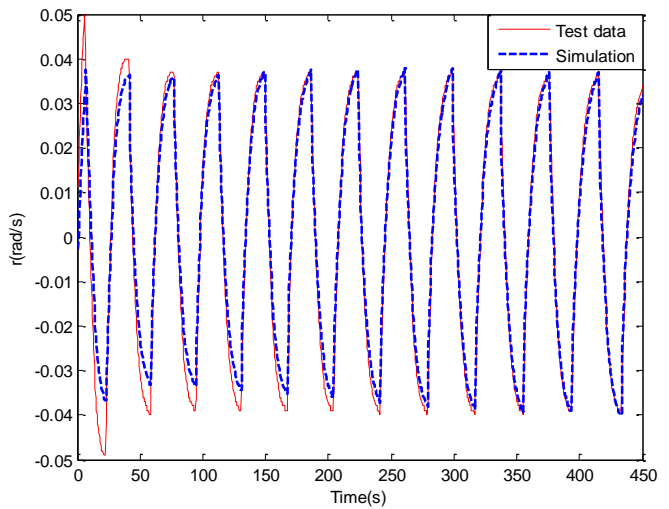

Figure 3. Time histories of the yaw rate

At the beginning of AUV maneuvering motion, the flow field around AUV changes tempestuously that makes the identified hydrodynamic coefficients have some error. The results of simulation are distorted at the beginning. With the AUV maneuvering motion stabilized, the flow field around AUV is also stabilized. The results of simulation are in good agreement with the model test data. In total, the identification results are close to test data, which verifies the validation of sliding window LS-SVM in on-line modeling of AUV maneuvering.

\section{THE COMPARISON OF SLIDING WINDOW LS-SVM AND INCREMENTAL LS-SVM}

Incremental LS-SVM has been proved to be an effective method of parametric identification. So its identified results can be used to demonstrate sliding window LS-SVM.

800 samples gathered by horizontal $10^{\circ} / 10^{\circ}$ zigzag test are taken as test data. The paper takes 400 of the data as training samples and the others as predicted samples. The results of the sliding window LS-SVM are compared with incremental LS-SVM results as shown in Fig.4 and Fig.5.

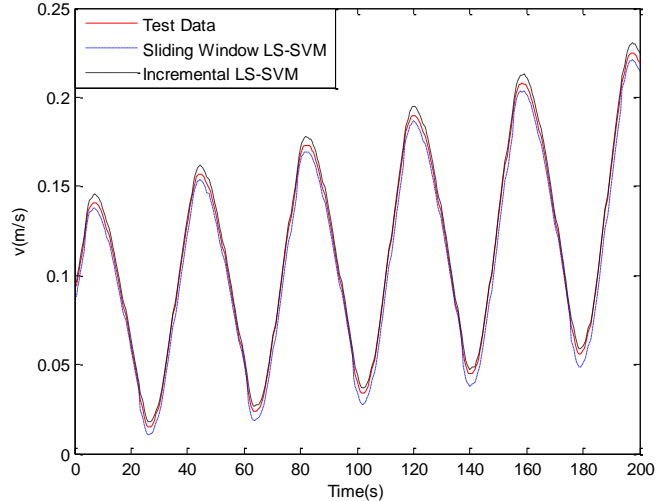

Figure 4. The predicted results of the transverse speed

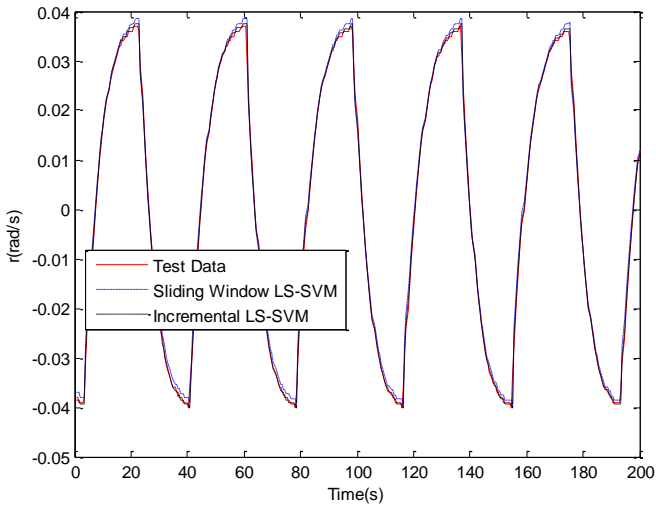

Figure 5. The predicted results of the yaw rate

To value the predicted performance, mean square root error is taken as the target.

$$
M S E=\sqrt{\frac{1}{M} \sum_{k=1}^{M}[y(k)-\hat{y}(k)]^{2}}
$$

Where, $M$ is the predicted sampling number, $y(k)$ is the experimental value, $\hat{y}(k)$ is the predicted value.

TABLE II. MSE OF THE TWO METHODS’ PREDICTION

\begin{tabular}{|c|c|c|}
\hline & $M S E$ of transverse speed $v$ & $M S E$ of yaw rate $r$ \\
\hline $\begin{array}{c}\text { Sliding window } \\
\text { LS-SVM }\end{array}$ & 0.0057 & 0.0016 \\
\hline Incremental \\
LS-SVM
\end{tabular}

With regarding to the on-line identification, we should increase the algorithm's computing speed and reduce the storage space. To demonstrate the superiority of sliding window LS-SVM in these aspects, different numbers of samples are trained by using sliding window LS-SVM and 
incremental LS-SVM. The use results of runtime (the total time that cost by training all the samples) and storage space (only calculate the storage space of main variables) are shown in Table III.

TABLE III. THE USE RESULTS OF RUNTIME AND STORAGE SPACE

\begin{tabular}{|c|c|c|c|c|}
\hline \multirow{2}{*}{$\begin{array}{c}\text { Number of } \\
\text { Samples }\end{array}$} & \multicolumn{2}{|c|}{ Runtime(s) } & \multicolumn{2}{c|}{ Storage space(Bytes) } \\
\cline { 2 - 5 } & Sliding & Incremental & $\begin{array}{c}\text { Sliding } \\
\text { window } \\
\text { LS-SVM }\end{array}$ & $\begin{array}{c}\text { Incremental } \\
\text { LS-SVM }\end{array}$ \\
\hline 200 & 0.2265 & 0.6595 & $1.88 \mathrm{E}+05$ & $3.26 \mathrm{E}+05$ \\
\hline 400 & 0.8012 & 1.4050 & $1.94 \mathrm{E}+05$ & $1.29 \mathrm{E}+06$ \\
\hline 600 & 1.4092 & 4.4514 & $1.99 \mathrm{E}+05$ & $2.90 \mathrm{E}+06$ \\
\hline 800 & 1.8811 & 10.1427 & $2.07 \mathrm{E}+05$ & $5.15 \mathrm{E}+06$ \\
\hline
\end{tabular}

From the analysis we know, compared to incremental LS-SVM, the calculation accuracy of sliding window LSSVM is close to incremental LS-SVM. But it costs less computing time and storage space.

\section{SUMMARY}

SVM is widely used to identify the parameters of system. But there exist some problems in on-line identification. Sliding window LS-SVM is deduced and applied for on-line modeling of AUV maneuvering. Then sliding window LSSVM is adopted to identify the Abkowitz model by using free-running test data and the veracity of the parameters identified is validated by using the system simulation. The simulation results demonstrate that the method can identify the parameters effectively. Compared to incremental LSSVM, Sliding window LS-SVM costs less computing time and storage space. Not only does this method supply an effective method to model AUV's maneuvering motion, but also provide a good technique for the motion simulation and the Maneuverability prediction.

\section{REFERENCES}

[1] Shi Shengda. Submarine Maneuverability(in Chinese)[M]. Beijing: National Industry Press, 1995.

[2] Luo Wei-lin, Zou Zao-jian. Identification of response models of ship maneuvering motion using Support Vector Machines[J]. Journal of Ship Mechanics, 2007, 11(6): 832-838.

[3] Luo Wei-lin, Zou Zao-jian. Prediction of ship maneuvering by using Support Vector Machines[C]. Workshop on Verification and Validation of Ship Maneuvering Simulation Methods. Copenhagen, Denmark: SIMMAN 2008, April 2008: Vol. 1, E28-E32.

[4] Luo Wei-lin, Zou Zao-jian. The Modeling of Ship Maneuvering Motion by using Least Square Support Vector Machines[J]. Journal of System Simulation, 2008,20(13): 3381-3384.

[5] Luo Wei-lin. On the Modeling of Ship Maneuvering Motion by using Support Vector Machines[D]. Shanghai: Shanghai Jiao Tong University, 2009.

[6] Xu Feng, Zou Zao-jian, Song Xin. Parametric Identification of AUV's Maneuvering Motion Based on Support Vector Machines[J]. Journal of Ship Mechanics, 2011, 15(9): 981-987.

[7] Xu Feng, Zou Zao-jian, Yin Jian-chuan. On-line Modeling of Ship
Maneuvering Motion Based on Support Vector Machines[J]. Journal of Ship Mechanics, 2012, 16(3):218-225.

[8] Suykens J A K, Vandewale J. Least squares support vector machine classifiers[J]. Neural Processing Letters, 1999, 9(3): 293-300.

[9] Zhang J K. Linear model's parameters estimation method and its improvement(in Chinese)[M]. Changsha: National University of Defense Technology Press, 1992.

[10] Fossen T I. Guidance and Control of Underwater Vehicles[M]. New York: John Wiley \& Sons, 1994.

[11] Li Ye, Liu Jiancheng, Shen Mingxue. Dynamics model of underwater robot motion control in 6 degrees of freedom[J]. Journal of Harbin Institute of Technology, 2005, 12(4): 456-459. 